\title{
Three-Dimensional Routing in Underwater Acoustic Sensor Networks
}

\author{
Dario Pompili and Tommaso Melodia \\ Broadband and Wireless Networking Laboratory \\ School of Electrical \& Computer Engineering \\ Georgia Institute of Technology, Atlanta, GA 30332 \\ \{dario, tommaso\}@ece.gatech.edu
}

\begin{abstract}
Underwater sensor networks will find applications in oceanographic data collection, pollution monitoring, offshore exploration, disaster prevention, assisted navigation, and tactical surveillance applications. In this paper, the problem of data gathering in a 3D underwater acoustic sensor network is investigated at the network layer, by considering the interactions between the routing functions and the characteristics of the underwater channel. Two routing algorithms are proposed for delay-insensitive and delay-sensitive sensor network applications, respectively.

The proposed distributed algorithm for delay-insensitive applications allows each node to select its next hop, with the objective of minimizing the energy consumption taking into account the varying condition of the underwater channel. A centralized algorithm for delay-sensitive routing in an underwater environment is proposed. The solution relies on topology information gathered by a surface station, which optimally configures the network paths. The proposed algorithms are shown to achieve the performance targets of the underwater environment by means of simulation.
\end{abstract}

Categories and Subject Descriptors:

C.2.2 [Computer-Communication Networks]: Network Protocolsrouting protocols

General Terms: Algorithms, Design, Reliability, Performance.

Keywords: Underwater Acoustic Sensor Networks, Routing Algorithms, Mathematical programming/Optimization.

\section{INTRODUCTION}

Underwater sensor networks [6] are envisioned to enable applications for oceanographic data collection, pollution monitoring, offshore exploration, disaster prevention, assisted navigation, and tactical surveillance applications. Wireless underwater acoustic networking is the enabling technology for these applications. UnderWater Acoustic Sensor Networks (UW-ASN) consist of sensors that are deployed to perform collaborative monitoring tasks over a given volume. To achieve this objective, sensors must be organized in an autonomous network that self-configures according to

Permission to make digital or hard copies of all or part of this work for personal or classroom use is granted without fee provided that copies are not made or distributed for profit or commercial advantage and that copies bear this notice and the full citation on the first page. To copy otherwise, to republish, to post on servers or to redistribute to lists, requires prior specific permission and/or a fee.

PE-WASUN'05, October 10-13, 2005, Montreal, Quebec, Canada.

Copyright 2005 ACM 1-59593-182-1/05/0010 ...\$5.00. the varying characteristics of the ocean environment. The above described features enable a broad range of applications:

- Ocean Sampling. Networks of sensors and AUVs (Autonomous Underwater Vehicles) can perform synoptic sampling.

- Environmental Monitoring. UW-ASN can perform pollution monitoring (chemical, biological, and nuclear).

- Disaster Prevention. Sensor networks that measure seismic activity from remote locations can provide tsunami warnings to coastal areas, or study the effects of seaquakes.

- Assisted Navigation. Sensors can be used to identify hazards on the seabed, and to locate dangerous rocks or shoals.

- Distributed Tactical Surveillance. AUVs and sensors can monitor areas for surveillance, reconnaissance, targeting, and intrusion detection.

- Mine Reconnaissance. AUVs can be used to perform rapid environmental assessment and detect mine-like objects.

Acoustic communications are the typical physical layer technology in underwater networks. In fact, radio waves propagate through conductive sea water only at extra low frequencies $(30-300 \mathrm{~Hz})$, which require large antennae and high transmission power. Optical waves do not suffer from such high attenuation but are affected by scattering. Thus, links in underwater networks are usually based on acoustic wireless communications [16].

Although there exist many recently developed network protocols for wireless sensor networks, the unique characteristics of the underwater acoustic communication channel [14] require very efficient and reliable new data communication protocols.

Major challenges in underwater acoustic networks are:

1. Propagation delay is five orders of magnitude higher than in radio frequency terrestrial channels and variable;

2. The underwater channel is severely impaired, especially due to multipath and fading problems;

3. The available bandwidth is severely limited;

4. High bit error rates and temporary losses of connectivity (shadow zones) can be experienced;

5. Sensors may fail because of fouling and corrosion;

6. Battery power is limited and usually batteries cannot be easily recharged, also because solar energy cannot be exploited. 
Most impairments of the underwater acoustic channel are adequately addressed at the physical layer, by designing receivers able to deal with high bit error rates, fading, and the inter-symbol interference (ISI) caused by multipath. Conversely, characteristics such as the extremely long and variable propagation delays are better addressed at higher layers. Finally, as in terrestrial sensor networks, energy conservation is one of the major concerns, since batteries cannot be easily recharged or replaced. Moreover, the bandwidth of the underwater links is severely limited. Hence, routing protocols for underwater networks must be extremely energy efficient.

For these reasons, we introduce a model that allows investigating some fundamental characteristics of the underwater environment. In particular, the model highlights the underwater channel utilization efficiency as a function of the distance between the corresponding nodes and of the packet size. Based on the insights provided by the model, we propose two new routing algorithms for the $3 \mathrm{D}$ underwater environment, designed to meet the requirements of delay-insensitive and delay-sensitive sensor network applications, respectively.

The first routing algorithm deals with delay-insensitive applications. The proposed algorithm allows achieving two apparently conflicting objectives, i.e., increasing the channel utilization efficiency without increasing the packet error rate. In a distributed fashion, the algorithm allows each node to select its best next hop with the objective of minimizing the energy consumption, taking into account the varying condition of the channel. The second algorithm is designed for delay-sensitive applications. It consists of a centralized solution, based on a network manager that resides on a surface station.

The remainder of this paper is organized as follows. In Section 2 , we discuss the suitability of the existing ad hoc and sensor routing solutions for the underwater environment. In Section 3, we discuss the underwater channel utilization, and compare it with the terrestrial radio channel. In Section 4, we introduce the network, propagation, and delay models that are used in the routing problem formulations. In Section 5, we introduce a distributed routing algorithm for delay-insensitive applications, while in Section 6, we propose a surface-assisted routing algorithm for delay-sensitive underwater applications. Finally, in Section 7 we show the performance results, and in Section 8 we draw the main conclusions.

\section{RELATED WORK}

In the last few years there has been an intensive study in routing protocols for ad hoc wireless networks [3] and sensor networks [5]. However, due to the different nature of the underwater environment and applications, there are several drawbacks with respect to the suitability of the existing solutions for underwater acoustic networks. The existing routing protocols are usually divided into three categories, namely proactive, reactive, and geographical routing protocols. Proactive protocols provoke a large signaling overhead to establish routes, since updated topology information has to be propagated to all the nodes in the network. This way, each node is able to establish a path to any other node in the network, which may not be needed in UW-ASNs. Reactive protocols are more suitable for dynamic environments but incur a higher latency and still require source-initiated flooding of control packets to establish paths. Reactive protocols are deemed to be unsuitable for UW-ASNs as they also cause a high latency in the establishment of paths, which may be amplified underwater by the slow propagation of acoustic signals. Geographical routing protocols are very promising for underwater communications because of their scalability feature and their limited required signaling. However, Global Positioning System (GPS) receivers do not work in underwater properly. For these

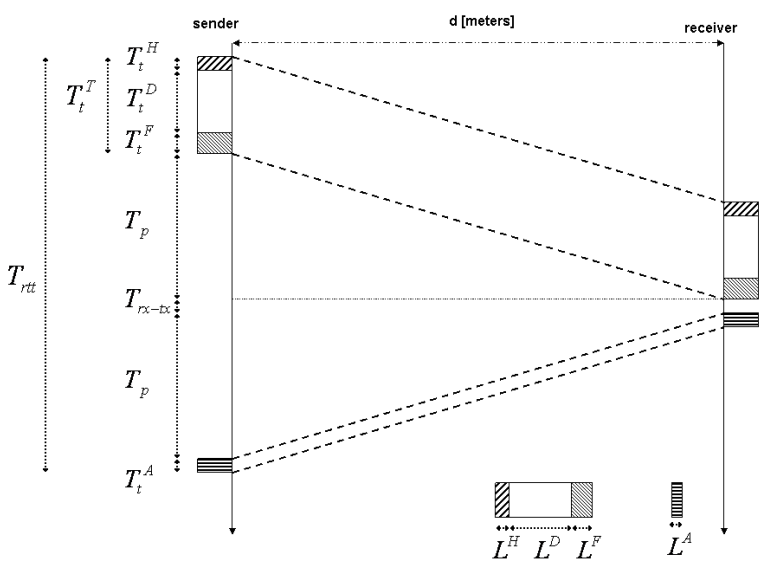

Figure 1: Underwater channel utilization efficiency

reasons, acoustic techniques to solve the localization problem with limited energy expenditure in the presence of measurement errors need to be further investigated.

Some recent papers propose network layer protocols specifically tailored to underwater acoustic networks. In [18], a routing protocol is proposed that autonomously establishes the underwater network topology, controls network resources, and establishes network flows, which relies on a centralized network manager running on a surface station. The manager establishes efficient data delivery paths in a centralized fashion, which allows avoiding congestion and providing some form of quality of service guarantee. Although the idea is promising, the performance evaluation of the proposed mechanisms has not been thoroughly studied yet. In [15] the authors provide a simple design example of a shallow water network, where routes are established by a central manager based on neighborhood information gathered from all nodes by means of poll packets. However, the paper does not describe routing issues in detail, i.e., it does not discuss the criteria used to select data paths. Moreover, sensors are only deployed linearly along a stretch, while the characteristics of the 3D underwater environment are not investigated.

\section{UNDERWATER CHANNEL EFFICIENCY}

In this section, we introduce an analytical model to study the effect of the characteristics of the underwater environment on the channel utilization efficiency when the medium is shared by several devices. The objective is to outline fundamental characteristics of the underwater environment, so as to provide guidelines for the design of routing solutions.

We consider a shared channel where a device transmits a data packet when it senses the channel idle, and the corresponding device advertises a correct reception with a short ACK packet. By referring to Fig. 1, we assume that the payload of the data packet to be transmitted has length $L^{D}$ bits, while the header has length $L^{H}$ bits. Moreover, the packet may be protected with a forward error correction (FEC) mechanism, which introduces a redundancy of $L^{F}$ bits. The ACK packet is assumed to be $L^{A}$ bits long. Given a transmission rate of $r$ bps, the round-trip transmission time is

$$
T_{r t t}=T_{t}^{H}+T_{t}^{D}+T_{t}^{F}+2 \cdot T_{p}+T_{r x-t x}+T_{t}^{A},
$$

where $T_{t}^{H}, T_{t}^{D}, T_{t}^{F}$, and $T_{t}^{A}$ are the transmission times of the header, payload, FEC overhead, and ACK packet, respectively, while 
$T_{p}$ is the propagation delay, and $T_{r x-t x}$ is the time needed to process the packet and switch the circuitry from receiving to transmitting mode. We define the channel utilization efficiency as

$$
\eta=\frac{\frac{L^{D}}{\hat{N}^{t} \cdot T_{r t t}}}{r}, \hat{N}^{t}=(1-P E R) \cdot \sum_{n=1}^{\infty} n \cdot P E R^{n-1} .
$$

where $\hat{N}^{t}$ represents the average number of transmissions for the receiver to successfully decode a packet, given a packet error rate (PER) on the link. We refer to the expression $\frac{L^{D}}{\hat{N}^{t} \cdot T_{r t t}}$ as effective link capacity between the sender and the receiver; it represents the average bit rate achievable by a contention-free medium access control scheme. Thus, the expression in (2) can be rewritten as

$$
\eta=\frac{L^{D}}{\hat{N}^{t} \cdot\left[L^{D}+L^{H}+L^{A}+L^{F}+r \cdot\left(2 \frac{d}{q}+T_{r x-t x}\right)\right]},
$$

where the propagation delay $T_{p}$ is expressed as the ratio between the distance between the sender and the receiver, and the speed $q$ of the signal in the medium.

Figures 2(a) and 2(b) show the channel efficiency (3) for an underwater environment, where we set the speed of sound in water to $q=1500 \mathrm{~m} / \mathrm{s}$ (see Section 4.3), and the transmission rate to $r=50 \mathrm{kbps}$. Figure 2(a) refers to transmissions without forward error correction (i.e., $L^{F}=0$ ), while Fig. 2(b) refers to a ReedSolomon FEC $(255,239)$, which is able to correct up to 8 bit errors in a block of 255 bits [13]. The bit error rate on the channel is assumed to be linearly increasing with decreasing signal-to-noise ratio (SNR) (between $10^{-2}$ and $10^{-6}[15]$ ), and errors are assumed to be uniformly distributed in time. The decaying law for the transmitted power in underwater will be discussed in detail in Section 4.2. The two figures consider a range of distances between 100 and 500 meters. As can be seen in Fig. 2(a), the maximum channel efficiency is 0.25 over a distance of $100 \mathrm{~m}$ with packet size equal to about $1 K B$ yte, while it drops below 0.05 for distances greater than $200 \mathrm{~m}$. When we apply FEC techniques in the same environment, a maximum channel utilization efficiency of 0.77 can be achieved over $100 \mathrm{~m}$ with packets of 5 KByte. The efficiency degrades abruptly with increasing distance, and the optimal packet size, i.e., the packet size that yields maximum channel utilization efficiency on a given distance, decreases as well. Larger packets tend to improve the channel efficiency; at the same time, given a bit error rate (BER), the packet error rate (PER) increases with increasing packet size, thus increasing the average number of transmissions for a single packer. Hence, the optimal packet size is determined as the equilibrium between these two contrasting phenomena.

Figures 2(c) and 3(a) show the same phenomena for a terrestrial radio channel, characterized by a propagation speed $q=3 \cdot 10^{8} \mathrm{~m} / \mathrm{s}$ and transmission rate $r=1 \mathrm{Mbps}$. The bit error rate on the channel is assumed to be linearly increasing with decreasing SNR (between $10^{-3}$ and $10^{-7}$ ). With respect to the underwater environment, the values of channel utilization efficiency are higher and degrade more smoothly with increasing distance. In general, the optimal packet sizes are much smaller with respect to the underwater case.

In Figs. 3(b) and 3(c) we show the channel utilization efficiency for shorter transmission distances, i.e., from 20 to 100 meters. The absolute values are much higher in this case, while the degradation with increasing distance is smoother with respect to Figs 2(a) and 2(b). Noticeably, the optimal packet sizes are much higher in this case, i.e., we can increase the utilization of the channel by transmitting long packets as the PER increases smoothly with increasing packet size over this range of distances. When a FEC technique is applied (Fig. 2(b)), the optimal packet size increases consistently.
To summarize, we observe the following facts in the underwater environment:

1. The channel efficiency is very low. This, combined with very low data rates, can be detrimental for underwater communications. Hence, it is crucial to maximize the efficiency in exploiting the available resources.

2. The channel efficiency drops abruptly with increasing distance, and with varying packet size. Hence, it is important to jointly consider the packet size and the relative positions of next-hop nodes when taking routing decisions. This will be a key criterion in the design of the distributed routing solution that will be introduced in Section 5 .

3. Underwater communications greatly benefit from the use of forward error correction mechanisms. In fact, FEC strategies can decrease the average number of transmissions $\hat{N}^{t}$. The increasing packet error rate on longer-range underwater links can be compensated by either decreasing the packet length, or by applying stronger FEC algorithms.

\section{UNDERWATER NETWORK MODELS}

In this section, we introduce the models that will be used in the formulation of our routing algorithms.

\subsection{Network Model}

The underwater network can be represented as a graph $\mathcal{G}(\mathcal{V}, \mathcal{E})$, where $\mathcal{V}=\left\{v_{1}, . ., v_{N}\right\}$ is a finite set of nodes (vertexes) in a finitedimension 3D space, with $N=|\mathcal{V}|$, and $\mathcal{E}$ is the set of links (edges) among nodes, i.e., $e_{i j}$ equals 1 if nodes $v_{i}$ and $v_{j}$ are within each other's transmission range. The node $v_{N}$ (also $N$ for simplicity) represents the sink, i.e., the surface station. Each link $e_{i j}$ is associated with its estimated mean propagation delay $\overline{p_{i j}}$, with the estimated standard deviation of the propagation delay, $\sigma_{i j}$, and with the estimated $\operatorname{cost} c_{i j}$, i.e., the energy consumption to transmit one bit over that link. All these values are dependent on the 3D positions of nodes $i$ and $j$, as discussed in Section 6.1. $D$ is the matrix whose element $d_{i j}$ describes the distance between nodes $v_{i}$ and $v_{j}$ (also $i$ and $j$ in the following). Let $\mathcal{S}$ be the set of traffic sources, with $N_{S}=|\mathcal{S}|$. This set represents the sensor nodes that sense information from the underwater environment and send it to the surface station.

\subsection{The Urick Propagation Model}

The transmission loss $T L(d, f)$ that a narrow-band acoustic signal centered at frequency $f$ experiences along a distance $d$ is measured in $d B$, and can be accurately described by the Urick propagation model [17],

$$
T L(d, f)=20 \cdot \log (d)+\alpha(f) \cdot d+A .
$$

In (4), $d$ is expressed in $m, f$ in $k H z$, while $\alpha(f)$, measured in $d B / m$, represents the medium absorption coefficient, which describes the dependency of the transmission loss on the frequency band. The quantity $A$, measured in $d B$, is the so-called transmission anomaly, and accounts for the degradation of the acoustic intensity caused by multiple path propagation, refraction, diffraction, and scattering of sound. Its value is higher for horizontal links (up to $10 d B$ ), which are more affected by multipath [17]. More details about the Urick propagation model can be found in the appendix.

\subsection{Underwater Sound Speed Model}

The underwater acoustic propagation speed is [17]

$$
q(z, S, t)=1449.05+45.7 \cdot t-5.21 \cdot t^{2}+0.23 \cdot t^{3}+
$$




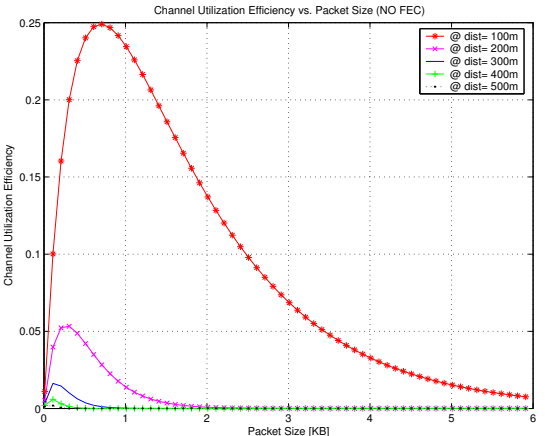

(a)

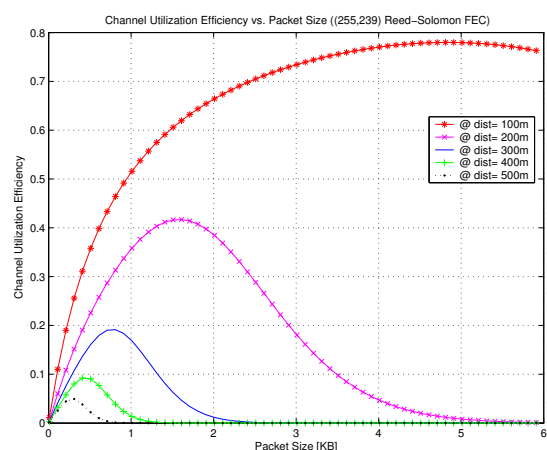

(b)

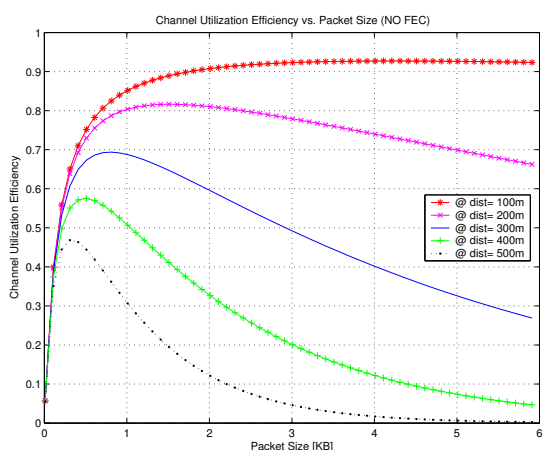

(c)

Figure 2: Underwater channel efficiency vs. packet size for different distances $(100 \mathrm{~m}-500 \mathrm{~m})$ without (a) and with Reed-Solomon FEC (b). Terrestrial channel efficiency vs. packet size without Reed-Solomon FEC (c)

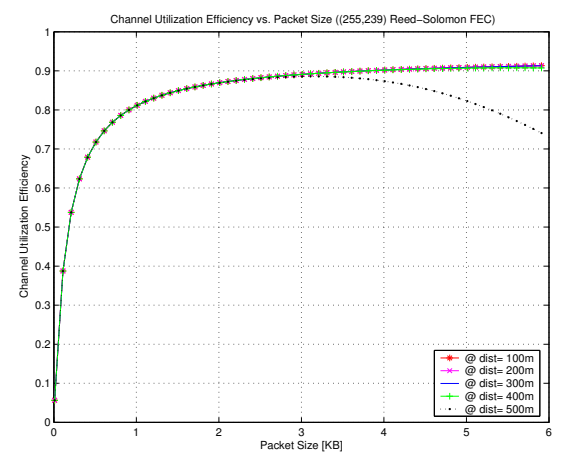

(a)

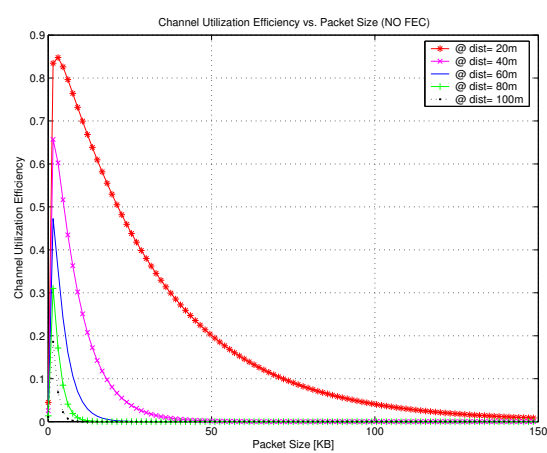

(b)

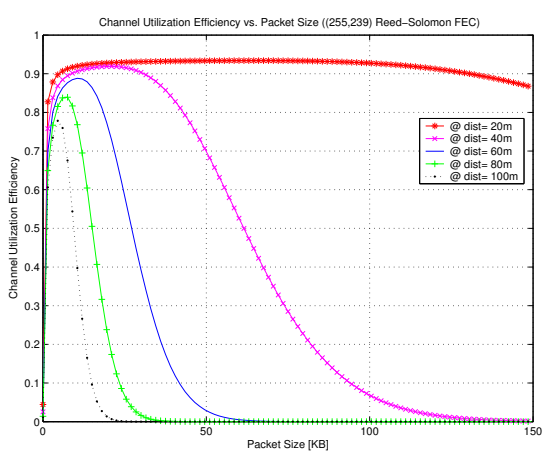

(c)

Figure 3: Terrestrial channel efficiency vs. packet size with Reed-Solomon FEC (a). Underwater channel utilization efficiency vs. packet size for different distances $(20 \mathrm{~m}-100 \mathrm{~m})$ without (b) and with Reed-Solomon FEC (c)

$+\left(1.333-0.126 \cdot t+0.009 \cdot t^{2}\right) \cdot(S-35)+16.3 \cdot z+0.18 \cdot z^{2}$

where $q(z, S, t)$ is in $m / s, t=T / 10$ (T is the temperature in ${ }^{\circ} C$ ), $S$ is the salinity in $p p t$, and $z$ is the depth in $k m$. The above expression provides a useful tool to determine the propagation speed in different operating conditions, and yields values centered around $1500 \mathrm{~m} / \mathrm{s}$.

\section{DELAY-INSENSITIVE ROUTING}

In this section, we introduce a distributed geographical routing solution for delay-insensitive applications in a 3D underwater environment. As discussed in Section 3, the objective of our proposed solution is to efficiently exploit the channel and to minimize the energy consumption. For this reason, we introduce the concept of packet train. A packet train is a juxtaposition of packets, which are transmitted back-to-back by a node without releasing the channel, in a single atomic transmission. The corresponding node sends an ACK for each train. The ACK can either cumulatively acknowledge the whole train, i.e., all the consecutively transmitted packets, or it can selectively request the retransmission of specific packets, which are then included in the next train. In Section 3, we discussed the existing trade-off between the channel utilization efficiency and the packet error rate, both increasing with increasing packet size. The strategy proposed here allows achieving two apparently conflicting objectives, i.e., increasing the efficiency of the channel by increasing the size of the transmitted train; and limiting the packet error rate by keeping the transmitted packets short. In other words, we decouple the effect of the packet length from the choice of the size of the train: while the former determines the packet error rate, the latter can be increased as needed to increase the channel utilization efficiency.

The proposed algorithm selects the next hop based on an energy metric. Furthermore, it tries to exploit those links that guarantee a low packet error rate, in order to maximize the probability that the packet is correctly decoded at the receiver. In other words, the energy efficiency of the link is weighted with the number of required retransmissions, with the ultimate objective of saving energy.

More formally, node $i$ will select $j^{*}$ as its next hop iff

$$
j^{*}=\operatorname{argmin}_{j \in \mathcal{S}_{i} \cap \mathcal{P}_{i}^{s}} E_{i}^{(j)},
$$

- $\mathcal{S}_{i}$ is the set of neighbors of node $i$, and $\mathcal{P}_{i}^{s}$ is the set of nodes closer to sink $s$ than node $i$, i.e., $j \in \mathcal{P}_{i}^{s}$ iff $d_{j s}<d_{i s}$,

and $E_{i}^{(j)}[J / b i t]$ is the following locally estimated link cost metric associated to link $(i, j)$, which represents an estimate of the energy required to successfully transmit a bit from node $i$ to the sink when $j$ is selected as next hop:

$$
E_{i}^{(j)}=E_{i j} \cdot \hat{N}_{i j}^{t} \cdot \hat{N}_{i}^{h o p} .
$$

The link cost metric in (7) is made up of the following three terms: 
1. $E_{i j}$ accounts for the energy to transmit one bit from node $i$ to node $j$. It can be expressed as

$$
E_{i j}=2 \cdot E_{\text {elec }}^{b}+\min \left(\frac{P_{T i}^{\max }}{r}, T L_{i j} \cdot E_{\text {min }}^{b}\right) .
$$

The meaning of the symbols is as follows:

- $E_{\text {elec }}^{b}[J / b i t]=E_{\text {elec }}^{\text {trans }}=E_{\text {elec }}^{\text {rec }}$, where $E_{\text {elec }}^{\text {trans }}$ and $E_{\text {elec }}^{r e c}$ are the energy per bit needed by transmitter electronics and digital processing, and by receiver electronics, respectively. These factors are independent of the distance.

- $P_{T i}^{\max }[W]$ is the maximum transmitting power of node $i$, while $r$ represents the transmission rate.

- $T L_{i j}$ is the transmission loss from $i$ to $j$, computed as in Section $4.2 ; E_{\text {min }}^{b}[J / b i t]$ represents the minimum amount of energy per bit required at the receiver to correctly decode the bit, given the noise level.

2. $\hat{N}_{i j}^{t}$ is the average number of transmissions of a packet of size $L[b i t]$ before it is correctly decoded at the receiver $j$, given a bit error rate on the link $(i, j)$. It can be expressed as

$$
\hat{N}_{i j}^{t}=\left(1-P E R_{i j}\right) \cdot \sum_{n=1}^{\infty} n \cdot P E R_{i j}^{n-1},
$$

where:

$$
P E R_{i j}=1-\sum_{k=0}^{C}\left(\begin{array}{l}
L \\
k
\end{array}\right) B E R_{i j}^{k} \cdot\left(1-B E R_{i j}\right)^{L-k},
$$

- $B E R_{i j}$ and $P E R_{i j}$ are the bit error rate and packet error rate of link $(i, j)$, respectively. The PER is calculated under the assumption that a FEC algorithm able to correct up to $C$ uniformly distributed errors is used at the receiver (if no FEC is used $C=0$ ).

3. $\hat{N}_{i}^{h o p}$ is the estimated number of hops from node $i$ to the surface station (sink), and is computed as

$$
\hat{N}_{i}^{h o p}=\max \left(\frac{d_{i s}}{<d_{i j}>_{i s}}, 1\right)
$$

- $d_{i j}$ is the distance between $i$ and $j$, while $<d_{i j}>_{i s}$ (which we refer to as advance) is the projection of $d_{i j}$ onto the line connecting node $i$ with the sink $s$.

Hence, the routing solution proposed for delay-insensitive applications allows node $i$ to select as next hop the node $j^{*}$ among its neighbors that satisfies the following requirements: $i)$ it is closer to the destination $s$; ii) minimizes the link metric $E_{i}^{(j)}$, which represents the energy required to successfully transmit a bit from node $i$ to the sink. The link metric in (7) takes into account the number of retransmissions $\left(\hat{N}_{i j}^{t}\right)$ associated to the link $(i, j)$, given the $B E R_{i j}$ and the packet size $L$. Moreover, it accounts for the average hop-path length $\left(\hat{N}_{i}^{h o p}\right)$, by assuming that the following hops will guarantee the same advance towards the surface station.

\section{DELAY-SENSITIVE ROUTING}

In this section, we introduce a routing solution for delay-sensitive applications in underwater sensor networks. As previously discussed, most recently developed routing protocols for terrestrial ad hoc and sensor networks are based on a packet switching paradigm, i.e., the routing function is performed separately for each single packet and paths are dynamically established.

Conversely, the characteristics of the underwater environment, along with the requirements of delay-sensitive applications, suggest to devise solutions based on some form of centralized planning of the network topology and of the data paths, in order to optimally exploit the extremely scarce network resources. For these reasons, virtual circuit routing techniques can be considered in UW-ASN for delay-sensitive applications, where multi-hop connections are established a priori between each source and sink, and each packet associated to a particular connection follows the same path. This requires a centralized coordination and implies a less flexible architecture, but allows exploiting powerful optimization tools on a centralized manager (e.g., the surface station) to achieve optimal performance at the network layer (such as minimum delay paths or energy efficient paths), with minimum signaling overhead.

Therefore, we propose a centralized formulation of the 3D routing problem for delay-sensitive applications. The objective of the optimization problem is to find two multi-hop node-disjoint data paths from each source to the surface station, namely primary and backup data paths, in order to offer protection against both node and link failures in the primary path. The protection scheme proposed in this section can be classified as a dedicated backup scheme with 1:1 path protection, with node-disjoint paths. Link protection schemes are not suitable for the underwater environment as they are too bandwidth consuming.

\subsection{Statistical Path Delay Model}

In this section, we model the delay of underwater links with the objective of statistically bounding the end-to-end delay. We model the propagation delay of each link $(i, j)$ as a random variable $p_{i j}$, with mean equal to $\overline{p_{i j}}$ and variance $\sigma_{i j}^{2}$. The mean $\overline{p_{i j}}=\tilde{d_{i j}} / \overline{q_{i j}}$ represents the average length of the multiple paths $\tilde{d_{i j}}$ divided by the the average underwater propagation speed of an acoustic wave propagating from node $i$ to node $j$. In vertical links sound rays propagate directly without bouncing on the bottom or surface of the ocean. Hence, the multi-path effect is negligible, and $\tilde{d_{i j}} \approx d_{i j}$. Conversely, for horizontal links, several rays propagate by bouncing on the bottom or surface of the ocean along with the direct ray. Hence, $\tilde{d_{i j}}$ is generally larger than $d_{i j}$. This is due to the fact that in state-of-the-art underwater receivers, multipath is compensated for by waiting for the energy associated to delayed rays. This way it is possible to exploit the energy spread on multiple paths. We further assume that the random variables modeling the link delays are statistically independent.

For each source $s \in \mathcal{S}$, we consider the binary variables $f_{i j}^{s}$ that equal 1 iff the link $(i, j)$ is part of the end-to-end path between source $s$ and the sink. Hence, we can express an upper bound to the end-to-end delay for source $s$ as $B^{s}=\sum_{(i, j) \in \mathcal{E}} p_{i j} \cdot f_{i j}^{s}$. Since the sum of Gaussian variables is itself a Gaussian variable, $B^{s}$ has a Gaussian distribution $B^{s} \backsim \mathcal{N}\left(\overline{B^{s}}, \sigma_{B^{s}}^{2}\right)$, where $\overline{B^{s}}=$ $\sum_{(i, j) \in \mathcal{E}} \overline{p_{i j}} \cdot f_{i j}^{s}$, and $\sigma_{B^{s}}^{2}=\sum_{(i, j) \in \mathcal{E}} \sigma_{i j}^{2} \cdot f_{i j}^{s}$. Thus, we can statistically bound the end-to-end delay $B^{s}$ of a path from source $s$ to the surface station, by imposing that the probability that $B^{s}$ exceeds the latency bound $B_{\max }$ be lower than a small value $\gamma$,

$$
\operatorname{Prob}\left\{B^{s} \geq B_{\max }\right\}=\frac{1}{2}\left[1-\operatorname{erf}\left(\frac{B_{\max }-\overline{B^{s}}}{\sqrt{2} \cdot \sigma_{B^{s}}}\right)\right] \leq \gamma
$$

where the $\operatorname{erf}$ function is defined as

$$
\operatorname{erf}(\Gamma)=\frac{2}{\sqrt{\pi}} \int_{0}^{\Gamma} e^{-t^{2} d t}
$$


This simplifies to $\overline{B^{s}}+\delta \cdot \sigma_{B^{s}} \leq B_{\max }$, where $\delta=\sqrt{2} \cdot \operatorname{erf}^{-1}(1-$ $2 \gamma$ ) only depends on $\gamma(\delta$ increases with decreasing values of $\gamma)$, which can be rewritten as,

$$
\sum_{(i, j) \in \mathcal{E}} \overline{p_{i j}} \cdot f_{i j}^{s}+\delta \cdot \sqrt{\sum_{(i, j) \in \mathcal{E}} \sigma_{i j}^{2} \cdot f_{i j}^{s}} \leq B_{\max }
$$

By expanding the second term of the sum in (14) with the Maclaurin series up to the first order, we obtain

$$
\sqrt{\sum_{(i, j) \in \mathcal{E}} \sigma_{i j}^{2} \cdot f_{i j}^{s}} \approx \sum_{(i, j) \in \mathcal{E}} f_{i j}^{s} \cdot\left(1+\frac{1}{2} \sigma_{i j}\right),
$$

which leads us to a linear constraint

$$
\sum_{(i, j) \in \mathcal{E}} f_{i j}^{s} \cdot\left[\overline{p_{i j}} \cdot+\delta \cdot\left(1+\frac{1}{2} \sigma_{i j}\right)\right] \leq B_{\max } .
$$

Equation (16) will be used in the formulation of the optimization problem in the following section.

\subsection{Integer Linear Programming Formulation}

We can formulate the problem of determining optimal primary and backup data paths for underwater sensor networks as an Integer Linear Program (ILP) [4]. We introduce the following notations:

- $e_{i j}$ is a binary variable representing a link that equals 1 iff $i$ and $j$ are within each other's transmission range;

- $c_{i j}$ is the cost of the link between nodes $i$ and $j$, i.e., the energy needed to transmit one bit;

- $f_{i j}^{1, s}$ is a binary variable that equals 1 iff link $(i, j)$ is in the primary path from the source $s$ to the surface station;

- $f_{i j}^{2, s}$ is a binary variable that equals 1 iff link $(i, j)$ is in the backup path from the source $s$ to the surface station;

- $\overline{p_{i j}}$ and $\sigma_{i j}^{2}$ are the mean and variance of the propagation delay for link $(i, j)$, respectively (see Section 6.1);

- $B_{\text {max }}$ is the maximum allowed delay for each source-to-sink path, to be respected with probability at least $1-\gamma$;

- $u_{i}$ is the capacity of node $i$ (number of concurrent flows, ingoing and outgoing, that it can handle);

- $l_{i j}$ is the capacity of link $(i, j)$ (number of concurrent flows that can be transmitted on the link).

The problem can be cast as follows: P: Optimal virtual connections for delay-sensitive underwater applications

$$
\begin{array}{cc}
\text { Given : } & e_{i j}, c_{i j}, \overline{p_{i j}}, \sigma_{i j}^{2}, B_{m a x}, w_{1}, w_{2}, u_{i}, l_{i j} \\
\text { Find: } & f_{i j}^{1, s}, f_{i j}^{2, s}
\end{array}
$$

Minimize: $\quad C^{T}=\sum_{s \in \mathcal{S}} \sum_{(i, j) \in \mathcal{E}} c_{i j} \cdot\left(w_{1} f_{i j}^{1, s}+w_{2} f_{i j}^{2, s}\right)$

Subject to :

$$
\begin{gathered}
\sum_{j \in \mathcal{V}}\left(f_{s j}^{x, s}-f_{j s}^{x, s}\right)=1, \forall s \in \mathcal{S}, \forall x=1,2 \\
\sum_{j \in \mathcal{V}}\left(f_{N j}^{x, s}-f_{j N}^{x, s}\right)=-1, \forall s \in \mathcal{S}, \forall x=1,2 \\
\sum_{j \in \mathcal{V}}\left(f_{i j}^{x, s}-f_{j i}^{x, s}\right)=0, \forall s \in \mathcal{S}, \forall i \in \mathcal{V}, i \neq s \text { and } i \neq N, \forall x=1,2
\end{gathered}
$$

$$
\begin{gathered}
f_{i j}^{x, s} \leq e_{i j}, \forall s \in \mathcal{S}, \forall i \in \mathcal{V}, \forall j \in \mathcal{V}, \forall x=1,2 \\
\sum_{s \in S}\left(f_{i j}^{1, s}+f_{i j}^{2, s}\right) \leq l_{i j}, \forall i \in \mathcal{V}, \forall j \in \mathcal{V} \\
\sum_{s \in S}\left[\sum_{j \in V}\left(f_{j i}^{1, s}+f_{j i}^{2, s}\right)+\sum_{j \in \mathcal{V}}\left(f_{i j}^{1, s}+f_{i j}^{2, s}\right)\right] \leq u_{i}, \forall i \in \mathcal{V} \\
f_{j i}^{1, s}+\sum_{n \in \mathcal{V}} f_{n i}^{2, s} \leq 1, \forall s \in \mathcal{S}, \forall i \in \mathcal{V} s . t . i \neq N, \forall j \in \mathcal{V} ; \\
\sum_{(i, j) \in \mathcal{E}} f_{i j}^{x, s} \cdot\left[\frac{123)}{p_{i j}}+\delta \cdot\left(1+\frac{1}{2} \sigma_{i j}\right)\right] \leq B_{\max }, \forall s \in \mathcal{S}, \forall x=1,2 .
\end{gathered}
$$

The objective function of problem $\mathbf{P}$ aims at minimizing the overall energy consumption as a sum of the energy consumptions of all links that compose the primary and backup data paths. Two different weights $w_{1}$ and $w_{2}$ are assigned to the primary and backup data paths, respectively, with $w_{1}+w_{2}=1$. Clearly, by increasing the relative weight $w_{2}$ we are increasing the weight of the backup paths in the optimal solution, i.e., we are trying to obtain energy efficient backup paths. This may worsen the energy consumption of the primary data paths, and should be done only in scenarios where we expect nodes to fail often, as will be discussed in Section 7. In general, we will have $w_{2}<<w_{1}$. Constraints (17), (18), and (19) express conservation of flows [4], i.e., each source generates a flow that has to reach the sink. In particular, constraint (17) imposes that a source node generates a flow, while non-source nodes do not generate any flow, for primary and backup data path, respectively. Constraint (18) requires that flows generated by each source be collected by the sink. Constraint (19) guarantees that the balance between incoming and outgoing flows be null for non-source and non-sink nodes. Constraint (20) forces data paths to be created on links between adjacent nodes (i.e., that are within transmission range of each other). Constraint (21) ensures that the sum of all flows (primary and backup) transported on a link do not exceed the link capacity, while constraint (22) imposes that the sum of all flows (incoming and outgoing, primary and backup) handled by a sensor node do not exceed the node capacity. Constraint (23) requires the primary and backup paths to be node disjoint. Finally, constraint (24) statistically bounds the delay of data paths, according to the model introduced in Section 6.1. It can be shown that problem $\mathbf{P}$ is at least as complex as the Geometric Connected Dominating Set problem, which is proven to be NP-complete [10]. However, it is still possible to solve $\mathbf{P}$ for networks of moderate size (up to 100 nodes), as UW-ASN are deemed to be.

\section{PERFORMANCE EVALUATION}

In Sections 7.1 and 7.2, we discuss the performance of the proposed routing algorithms for delay-insensitive and delay-sensitive applications, respectively.

\subsection{Routing for Delay-Insensitive Applications}

We extended the wireless package of the J-Sim Simulator [1], which implements the whole protocol stack of a sensor node, in order to simulate the characteristics of the underwater environment, as discussed in Section 4. In particular, we modeled the underwater transmission loss, the propagation delay, and the physical layer characteristics of underwater receivers. 
As far as the MAC layer is concerned, since the development of a new multiple access technique for the underwater environment is out of the scope of this paper and left for future work, we adapted the behavior of the IEEE 802.11 to the underwater environment. Firstly, we removed the RTS/CTS handshaking, as it yields unacceptable delays in a low-bandwidth high-delay environment. Secondly, we tuned all the parameters of the IEEE 802.11 according to the physical layer characteristics. For example, the value of the slot time in the 802.11 backoff mechanism has to account for the propagation delay at the physical layer [7]. Hence, while it is set to $20 \mu \mathrm{s}$ for 802.11 DSSS (Direct Sequence Spread Spectrum), we found that a value of $0.18 s$ is needed to allow devices a few hundred meters apart to share the underwater medium. This implies that the delay introduced by the backoff contention mechanism is several orders of magnitude higher than in terrestrial channels, which in turn leads to very low channel utilizations, as also previously discussed in Section 3. Moreover, we set the values of the contention windows $C W_{\min }$ and $C W_{\max }$ [7] to 4 and 16, respectively, whereas in 802.11 DSSS they are set to 32 and 1024 .

In the experiments, we considered 100 sensors randomly deployed in a volume of $100 \times 100 \times 100 \mathrm{~m}^{3}$. We set the transmission range to $70 \mathrm{~m}$, the bandwidth to $50 \mathrm{kbps}$, and the maximum transmission power to $1 W$.In Fig. 4(a) we show the packet delivery ratio for the Greedy Routing Scheme (GRS) [8], which sends the packet to the neighbor that is the closest to the destination, and for the distributed 3D routing algorithm, proposed in Section 5. While for both routing algorithms the packet delivery ratio decreases as the number of sources increases, i.e., when the data traffic increases, our solution significantly outperforms the greedy scheme. This happens because our solution selects those links that are characterized by a lower BER, and a higher energy efficiency.

\subsection{Routing for Delay-Sensitive Applications}

The optimization problem presented in Section 6.2 was implemented in AMPL [9], and solved with CPLEX [2], which uses a branch and bound algorithm to solve mixed integer linear problems. The results obtained by solving problem $\mathbf{P}$ are compared in this section with a simpler solution, where two node-disjoint shortest weighted paths are calculated with an energy metric. In the experiments, we considered 60 sensors randomly deployed in a volume of $100 \times 100 \times 100 \mathrm{~m}^{3}$. The maximum allowed delay $\boldsymbol{B}_{\max }$ is set to $3 s$.

Figure 4(b) shows the expected energy consumption of the network by weighting the cost of the primary and backup paths with the probability of using each of them. We assumed a Poissonian model to capture the reliability of each sensor node, by assuming a failure rate $\lambda=1 / 2$ year $^{-1}$. The expected energy consumption increases with the observation time, and decreases with increasing $w_{2}$. This happens because by increasing $w_{2}$ the objective function of problem $\mathbf{P}$ weights more the backup paths. Hence, when failures occur, the connections are switched to backup paths characterized by lower energy consumption, which ultimately results in decreased energy consumption. This phenomenon becomes more evident with increasing observation time.

Figure 4(c) shows the source block probability with increasing observation time and weight $w_{2}$. The source block probability is defined as the probability that a source is not able to transmit either on the primary or on the backup path, since both have at least one failed node. While the source block probability increases with increasing observation time, it depends only slightly on the weight $w_{2}$, which allows selecting $w_{2}$ mostly based on energy considerations, irrespective of the required reliability.

Figure 5(a) shows the average path delay for primary and backup paths for our solution and for the shortest paths. As can be easily seen, primary paths have lower average delays than backup paths. This can be explained by considering the fact that primary paths are more likely than backup paths to use vertical links to reach the surface station. As discussed in Section 4.3, vertical links suffer less from multipath and, thus, are characterized by lower delays.

Figure 5(b) shows a comparison of the average number of hops of source-to-sink connections on primary and backup paths. In general, primary paths are shown to be longer (higher number of hops). Since primary paths are also more energy efficient than backup paths, it can be concluded that multi-hop paths are favored by the energy model used, i.e., as in terrestrial wireless networks it is possible to save energy by relaying data through intermediate nodes.

Figure 5(c) compares our solution to primary and backup nodedisjoint shortest weighted paths calculated with a hop distance metric. It is interesting to notice that, while the number of hops of the paths calculated by our solution is doubled, the energy consumption is drastically lower than with a shortest number of hop metric. This corroborates our remark that multi-hop paths save energy in underwater communications.

\section{CONCLUSIONS}

In this paper, the problem of data gathering in a 3D underwater acoustic sensor networks was investigated at the network layer, by considering the interactions between the routing functions and the characteristics of the underwater channel. Two routing algorithms were proposed for delay-insensitive and delay-sensitive sensor network applications, respectively. The distributed algorithm for delay-insensitive applications allows each node to select its next hop, with the objective of minimizing the energy consumption taking into account the varying condition of the underwater channel. The centralized algorithm for delay-sensitive routing relies on topology information gathered by a surface station, which optimally configures the network paths. An online distributed pathrepair algorithm guarantees survivability of the network to node and link failures, by locally healing paths in case of disconnections or failures. The proposed algorithms were shown to achieve the performance targets of the underwater environment by means of simulation.

\section{Acknowledgments}

This work was supported by the Office of Naval Research under contract N00014-02-1-0564.

\section{REFERENCES}

[1] The J-Sim Simulator.

[2] CPLEX solver, http://www.cplex.com.

[3] M. Abolhasan, T. Wysocki, and E. Dutkiewicz. A review of routing protocols for mobile ad hoc networks. Journal of Ad Hoc Networks (Elsevier), 2:1-22, Jan. 2004.

[4] R. K. Ahuja, T. L. Magnanti, and J. B. Orlin. Network Flows: Theory, Algorithms, and Applications. Prentice Hall, February 1993.

[5] K. Akkaya and M. Younis. A survey on routing protocols for wireless sensor networks. Ad Hoc Networks (Elsevier), 3(3):325-349, May 2005.

[6] I. F. Akyildiz, D. Pompili, and T. Melodia. Underwater acoustic sensor networks: Research challenges. Ad Hoc Networks (Elsevier), 3(3):257-279, May 2005.

[7] G. Bianchi. Performance analysis of the IEEE 802.11 DCF. IEEE Journal on Selected Areas of Communications (JSAC), 18(3):535-547, Mar. 2000.

[8] G. Finn. Routing and addressing problems in large metropolitan-scale internetworks. Technical report, ISI res. rep ISU/RR- 87-180, March 1987.

[9] R. Fourer, D. M. Gay, and B. W. Kernighan. AMPL: A Modeling Language for Mathematical Programming. Duxbury Press / Brooks/Cole Publishing Company, 2002.

[10] M. R. Garey and D. S. Johnson. Computer and Intractability. W. H. Freeman and Co., 1979. 


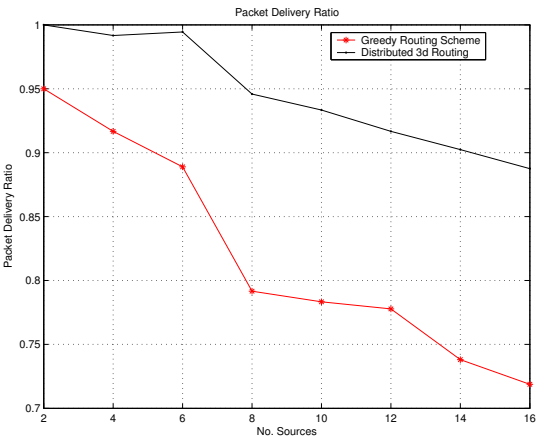

(a)

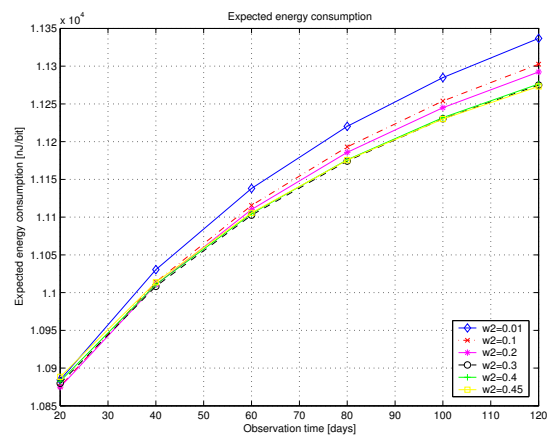

(b)

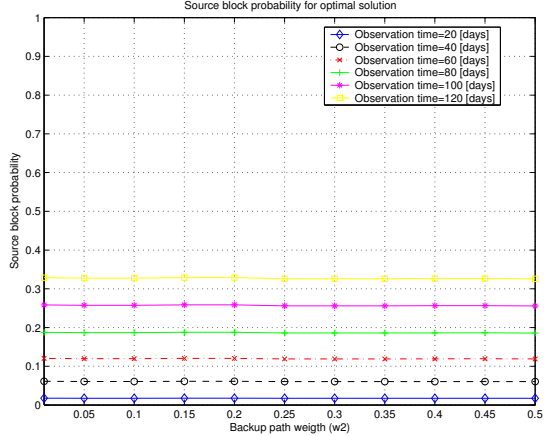

(c)

Figure 4: Packet delivery ratio for a greedy routing scheme and the distributed 3D routing (a). Expected energy consumption for primary and backup paths (b) and source block probability (c) for the centralized 3D routing

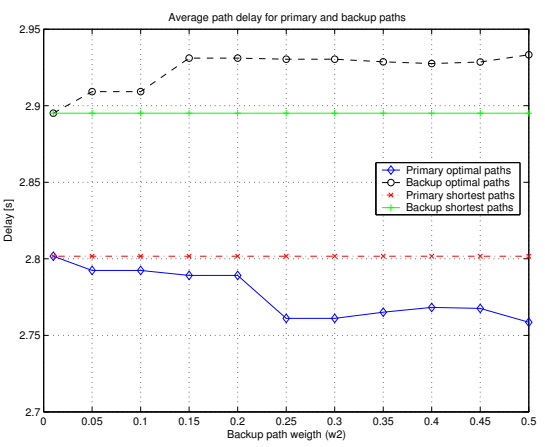

(a)

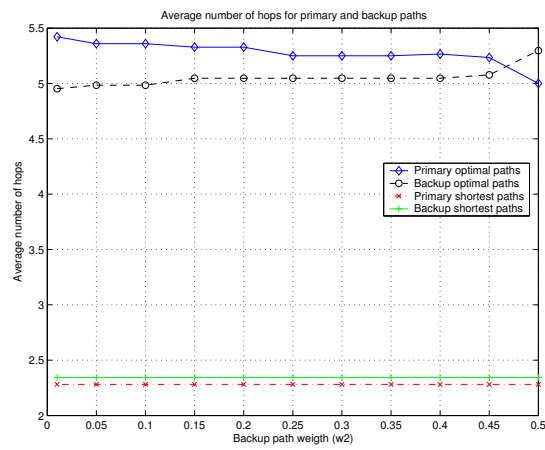

(b)

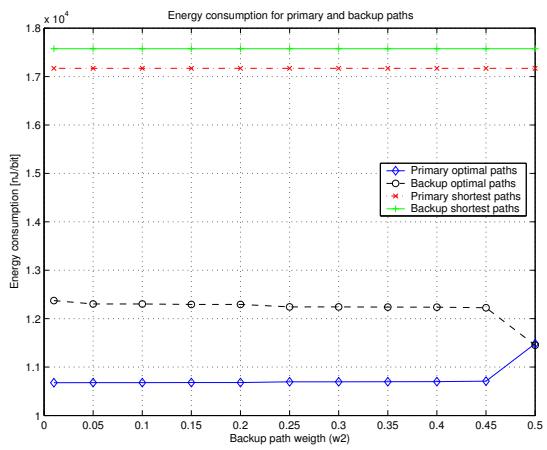

(c)

Figure 5: Comparison of average path delay (a) and average number of hops (b) for primary and backup paths (optimal and shortest path). Energy consumption for primary and backup path (optimal and minimum-hop path) (c)

[11] S. A. L. Glegg, R. Pirie, and A. LaVigne. A study of ambient noise in shallow water. In Florida Atlantic University Technical Report, 2000.

[12] R. Jurdak, C. V. Lopes, and P. Baldi. Battery lifetime estimation and optimization for underwater sensor networks. IEEE Sensor Network Operations, Winter 2004

[13] J. Proakis. Digital Communications. McGraw-Hill, New York, 1995.

[14] J. G. Proakis, E. M. Sozer, J. A. Rice, and M. Stojanovic. Shallow water acoustic networks. IEEE Communications Magazine, pages 114-119, Nov. 2001.

[15] E. M. Sozer, M. Stojanovic, and J. G. Proakis. Underwater acoustic networks IEEE Journal of Oceanic Engineering, 25(1):72-83, Jan. 2000.

[16] M. Stojanovic. Acoustic (underwater) communications. In J. G. Proakis, editor, Encyclopedia of Telecommunications. John Wiley and Sons, 2003.

[17] R. J. Urick. Principles of Underwater Sound. McGraw-Hill, 1983.

[18] G. Xie and J. H. Gibson. A network layer protocol for UANs to address propagation delay induced performance limitations. In Proceedings of IEEE OCEANS'01, volume 4, pages 2087-2094, Honolulu, HI, Nov. 2001.

\section{APPENDIX}

In this appendix, we provide more details on the Urick propagation model presented in Section 4.2.Different expressions of the medium absorption coefficient are available in the literature. It can be expressed as

$$
\alpha(f)=\alpha_{1}(f)+\alpha_{2}(f)+\alpha_{3}(f),
$$

where

$$
\begin{gathered}
\alpha_{1}(f)=\left[2.1 \cdot 10^{-10} \cdot(T-38)^{2}+1.3 \cdot 10^{-7}\right] \cdot f^{2}, \\
\alpha_{2}(f)=\frac{b \cdot f_{0}}{1+\left(\frac{f_{0}}{f}\right)^{2}}, b=2 \cdot S \cdot 10^{-5}, f_{0}=50 \cdot(T+1),
\end{gathered}
$$

and

$$
\alpha_{3}(f)=\frac{c \cdot f_{1}}{1+\left(\frac{f_{1}}{f}\right)^{2}}, c=1.2 \cdot 10^{-4}, f_{1}=\frac{10 \cdot(T-4)}{100} .
$$

In the above expressions, $T$ represents the temperature in ${ }^{\circ} C$, while $S$ is the water salinity in $p p t$ (parts per thousands). The overall absorption due to viscous friction and $\mathrm{MgSO}_{4}$ / boric acid relaxation is given in (25).

Considering that the sound from an acoustic source spreads omnidirectionally, the source level $S L$ is

$$
S L=10 \cdot \log \left(\frac{P / A_{1}}{I_{0}}\right)=10 \cdot \log (P)+171 d B_{\mu P a},
$$

where $A_{1}$ and $I_{0}$ represent the area measured one meter away from the source and the standard intensity $\left(6.504 \cdot 10^{-19} \mathrm{~W} / \mathrm{m}^{2}\right)$, respectively. Therefore, the signal-to-noise ratio at the receiver can be expressed by the Passive Sonar Equation [12],

$$
S N R=S L-T L-(N L-D I),
$$

where $S L$ is the source level, $T L$ is the transmission loss, $N L$ is the noise level, and $D I$ is the microphone directivity. Several studies of shallow water noise measurements [11] suggest considering an average value $N L$ of $70 d B_{\mu P a}$ for the ambient noise level, and a target $S N R$ of $15 d B$ at the receiver [12].

Since each device is characterized by a maximum transmission power $P_{T}^{m a x}$, the above model also defines the transmission range $R_{T}$ as the maximum distance between two sensors for successful transmission, given the minimum $S N R$ needed at the receiver to achieve a certain bit error rate. 